\title{
Towards a Sampled-Data Theory for Nonlinear Model Predictive Control
}

\author{
Rolf Findeisen ${ }^{1}$, Lars Imsland ${ }^{2}$, Frank Allgöwer ${ }^{1}$, and Bjarne Foss ${ }^{2}$ \\ 1 Institute for Systems Theory in Engineering, University of Stuttgart, \\ Pfaffenwaldring 9, D-70550 Stuttgart, Germany \\ \{findeise, allgower\}@ist.uni-stuttgart.de \\ 2 Department of Engineering Cybernetics, NTNU, 7491 Trondheim, Norway \\ \{Lars.Imsland,Bjarne.Foss\}@itk.ntnu.no
}

Summary. This paper considers the stability, robustness and output feedback problem for sampled-data nonlinear model predictive control (NMPC). Sampleddata NMPC here refers to the repeated application of input trajectories that are obtained from the solution of an open-loop optimal control problem at discrete sampling instants. Specifically we show that, under the assumption that the value function is continuous, sampled-data NMPC possesses some inherent robustness properties. The derived robustness results have a series of direct implications. For example, they underpin the intuition that small errors in the optimal input trajectory, e.g. resulting from an approximate numerical solution, can be tolerated. Furthermore, the robustness can be utilized to design observer-based semi-globally stable output feedback NMPC schemes.

\section{Introduction}

Model predictive control (MPC), also known as receding horizon control or moving horizon control, is by now a well established control method. Especially linear MPC, i.e. predictive control for linear systems considering linear constraints, is widely used in industry; mainly since it allows to handle MIMO systems and constraints on states and inputs systematically [38]. Motivated by the success of linear MPC, predictive control of nonlinear systems (NMPC) has gained significant interest over the past decade. Various NMPC strategies that lead to stability of the closed-loop have been developed in recent years and key questions such as the efficient solution of the occurring open-loop optimal control problem have been extensively studied (see e.g. [1,10,33] for recent reviews).

In this paper we are interested in stability, robustness, and output feedback for continuous time NMPC with sampled measurement information; i.e. we consider the stabilization of continuous time systems by repeatedly applying input trajectories that are obtained from the solution of an open-loop optimal 
control problem at discrete sampling instants. In the following we refer to this problem as sampled-data NMPC.

In the first part of this paper we briefly review how nominal stability for sampled-data NMPC can be achieved. Based on the nominal stability results we show in Section 4 that, under the assumption that the value function is continuous, the closed-loop using a nominally stable sampled-data NMPC scheme possesses some inherent robustness properties. Some consequences of the result are outlined. Expanding the robustness results obtained in Section 4 to measurement errors, we consider in Section 5 the output feedback problem for sampled-data NMPC. Specifically we state conditions on the observer error that must be satisfied to achieve semi-global practical stability of the closedloop.

\section{State Feedback Sampled-data NMPC}

We consider the stabilization of time-invariant nonlinear systems of the form

$$
\dot{x}(t)=f(x(t), u(t)), \quad x(0)=x_{0}
$$

subject to the input and state constraints: $u(t) \in \mathcal{U} \subset \mathbb{R}^{m}, x(t) \in \mathcal{X} \subseteq \mathbb{R}^{n}, \forall t \geq$ 0 . With respect to the vector field $f: \mathbb{R}^{n} \times \mathbb{R}^{m} \rightarrow \mathbb{R}^{n}$ we assume that it is locally Lipschitz continuous and satisfies $f(0,0)=0$. Furthermore, the set $\mathcal{U} \subset \mathbb{R}^{m}$ is compact, $\mathcal{X} \subseteq \mathbb{R}^{n}$ is connected, and $(0,0) \in \mathcal{X} \times \mathcal{U}$.

In sampled-data NMPC an open-loop optimal control problem is solved at discrete sampling instants $t_{i}$ based on the current state information $x\left(t_{i}\right)$. The sampling instants $t_{i}$ are given by a partition $\pi$ of the time axis.

Definition 1. (Partition) Every series $\pi=\left(t_{i}\right), i \in \mathbb{N}$ of (finite) positive real numbers such that $t_{0}=0, t_{i}<t_{i+1}$ and $t_{i} \rightarrow \infty$ for $i \rightarrow \infty$ is called $a$ partition. Furthermore, let $\bar{\pi}:=\sup _{i \in \mathbb{N}}\left(t_{i+1}-t_{i}\right)$ be the upper diameter of $\pi$ and $\underline{\pi}:=\inf _{i \in \mathbb{N}}\left(t_{i+1}-t_{i}\right)$ be the lower diameter of $\pi$.

When the time $t$ and $t_{i}$ occurs in the same setting, $t_{i}$ should be taken as the closest previous sampling instant $t_{i}<t$.

In sampled-data NMPC, the input trajectory applied in between the sampling instants is given by the solution of the following open-loop optimal control problem:

$$
\begin{array}{ll}
\min _{\bar{u}(\cdot)} J\left(\bar{u}(\cdot) ; x\left(t_{i}\right)\right) \text { subject to: } \quad & \dot{\bar{x}}(\tau)=f(\bar{x}(\tau), \bar{u}(\tau)), \quad \bar{x}\left(t_{i}\right)=x\left(t_{i}\right) \\
& \bar{u}(\tau) \in \mathcal{U}, \quad \bar{x}(\tau) \in \mathcal{X} \quad \tau \in\left[t_{i}, t_{i}+T_{p}\right] \\
& \bar{x}\left(t_{i}+T_{p}\right) \in \mathcal{E} .
\end{array}
$$

The bar denotes predicted variables, i.e. $\bar{x}(\cdot)$ is the solution of (2a) driven by the input $\bar{u}(\cdot):\left[t_{i}, t_{i}+T_{p}\right] \rightarrow \mathcal{U}$ with the initial condition $x\left(t_{i}\right)$. The cost functional $J$ minimized over the control horizon $T_{p} \geq \bar{\pi}>0$ is given by 


$$
J\left(\bar{u}(\cdot) ; x\left(t_{i}\right)\right):=\int_{t_{i}}^{t_{i}+T_{p}} F(\bar{x}(\tau), \bar{u}(\tau)) d \tau+E\left(\bar{x}\left(t_{i}+T_{p}\right)\right),
$$

where the stage cost $F: \mathcal{X} \times \mathcal{U} \rightarrow \mathbb{X}$ is assumed to be continuous, satisfies $F(0,0)=0$, and lower bounded by a class $\mathcal{K}$ function ${ }^{3} \alpha_{F}: \alpha_{F}(\|x\|) \leq$ $F(x, u) \forall(x, u) \in \mathcal{X} \times \mathcal{U}$, where $\|\cdot\|$ denotes the Euclidean vector norm. The terminal region constraint $\mathcal{E}$ and the terminal penalty term $E$ are often used to enforce stability of the closed-loop $[20,33]$. The solution of the optimal control problem $(2)$ is denoted by $\bar{u}^{\star}\left(\cdot ; x\left(t_{i}\right)\right)$. It defines the open-loop input that is applied to the system until the next sampling instant $t_{i+1}$ :

$$
u\left(t ; x\left(t_{i}\right)\right)=\bar{u}^{\star}\left(t ; x\left(t_{i}\right)\right), \quad t \in\left[t_{i}, t_{i+1}\right) .
$$

The control $u\left(t ; x\left(t_{i}\right)\right)$ is a feedback, since it is recalculated at each sampling instant using the new state measurement.

Remark 1. The main idea behind predictive control is to solve the optimal control problem for the current state on-line. Thus, no explicit expression for $u^{\star}\left(t ; x\left(t_{i}\right)\right)$ is obtained. Note that this is not equivalent to the rather difficult task of finding a solution to the underlying Hamilton Jacobi Bellman PDE, since only the current state is considered. Typically the resulting dynamic optimization problem is solved using the so called direct approach, which has attracted significant research interest in recent years (see e.g. $[2,4,11,13,17$, $40,41])$. Specifically, it has been established that an on-line solution is possible for realistically sized problems even with present-day computing power.

We denote the solution of (1) starting at time $t_{1}$ from an initial state $x\left(t_{1}\right)$, applying an input $u:\left[t_{1}, t_{2}\right] \rightarrow \mathbb{R}^{m}$ by $x\left(\tau ; u(\cdot), x\left(t_{1}\right)\right), \tau \in\left[t_{1}, t_{2}\right]$. For clarity of presentation we limit ourselves to input signals that are piecewise continuous and thus refer to an admissible input as:

Definition 2. (Admissible Input) An input $u:\left[0, T_{p}\right] \rightarrow \mathbb{R}^{m}$ for a state $x_{0}$ is called admissible, if it is: a) piecewise continuous, b) $u(\tau) \in \mathcal{U} \forall \tau \in\left[0, T_{p}\right]$, c) $x\left(\tau ; u(\cdot), x_{0}\right) \in \mathcal{X} \forall \tau \in\left[0, T_{p}\right]$, d) $x\left(T_{p} ; u(\cdot), x_{0}\right) \in \mathcal{E}$.

Furthermore, we often refer to the so-called value function:

Definition 3. (Value function) The value function $V(x)$ is defined as the minimal value of the cost for the state $x: V(x)=J\left(\bar{u}^{\star}(\cdot ; x) ; x\right)$.

Various sampled-data NMPC schemes that guarantee stability and fit into the given setup exist $[6,7,16,20,25,31,36]$. These schemes differ in the way the terminal penalty term $E$ and (if it appears at all) the terminal region $\mathcal{E}$ are determined.

We do not assume an explicit controllability assumption on the system. Instead, as often done in NMPC, we derive stability under the assumption of initial feasibility of the optimal control problem.

\footnotetext{
${ }^{3}$ A continuous function $\alpha:[0, \infty) \rightarrow[0, \infty)$ is a class $\mathcal{K}$ function, if it is strictly increasing and $\alpha(0)=0$.
} 


\section{Nominal Stability of State Feedback NMPC}

The following theorem establishes conditions for the convergence of the closedloop states to the origin. It is a slight modification of the results given in [20] and $[5,6]$. We state it here together with a condensed proof, since it lays the basis for the robustness considerations in Section 4.

Theorem 1. (Stability of sampled-data NMPC) Suppose that

a) the terminal region $\mathcal{E} \subseteq \mathcal{X}$ is closed with $0 \in \mathcal{E}$ and that the terminal penalty $E(x) \in C^{1}$ is positive semi-definite

(b) $\forall x \in \mathcal{E}$ there exists an (admissible) input $u_{\mathcal{E}}:[0, \bar{\pi}] \rightarrow \mathcal{U}$ such that $x(\tau) \in \mathcal{E}$ and

$$
\frac{\partial E}{\partial x} f\left(x(\tau), u_{\mathcal{E}}(\tau)\right)+F\left(x(\tau), u_{\mathcal{E}}(\tau)\right) \leq 0 \quad \forall \tau \in[0, \bar{\pi}]
$$

(c) the NMPC open-loop optimal control problem is feasible for $t=0$.

Then for the closed-loop system (1), (4) $x(t) \rightarrow 0$ for $t \rightarrow \infty$, and the region of attraction $\mathcal{R}$ consists of the states for which an admissible input exists.

Proof. As usual in predictive control the proof consists of two parts: in the first part it is established that initial feasibility implies feasibility afterwards. Based on this result it is then shown that the state converges to the origin. Feasibility: Consider any sampling instant $t_{i}$ for which a solution exists (e.g. $\left.t_{0}\right)$. In between $t_{i}$ and $t_{i+1}$ the optimal input $\bar{u}^{\star}\left(\tau ; x\left(t_{i}\right)\right)$ is implemented. Since no model plant mismatch nor disturbances are present, $x\left(t_{i+1}\right)=$ $\bar{x}\left(t_{i+1} ; \bar{u}^{\star}\left(\tau ; x\left(t_{i}\right)\right), x\left(t_{i}\right)\right)$. Thus, the remaining piece of the optimal input $\bar{u}^{\star}\left(\tau ; x\left(t_{i}\right)\right), \tau \in\left[t_{i+1}, t_{i}+T_{p}\right]$ satisfies the state and input constraints. Furthermore, $\bar{x}\left(t_{i}+T_{p} ; x\left(t_{i}\right), \bar{u}^{\star}\left(\tau ; x\left(t_{i}\right)\right)\right) \in \mathcal{E}$ and we know from assumption (b) of the theorem that for all $x \in \mathcal{E}$ there exists at least one input $u_{\mathcal{E}}(\cdot)$ that renders $\mathcal{E}$ invariant on $\left[t_{i}+T_{p}, t_{i}+T_{p}+\bar{\pi}\right]$. Picking any such input we obtain as admissible input for any time $t_{i}+\sigma, \sigma \in\left(0, t_{i+1}-t_{i}\right]$

$$
\tilde{u}\left(\tau ; x\left(t_{i}+\sigma\right)\right)=\left\{\begin{array}{l}
\bar{u}^{\star}\left(\tau ; x\left(t_{i}\right)\right), \tau \in\left[t_{i}+\sigma, t_{i}+T_{p}\right] \\
u_{\mathcal{E}}\left(\tau-t_{i}-T_{p}\right), \tau \in\left(t_{i}+T_{p}, t_{i}+T_{p}+\sigma\right]
\end{array} .\right.
$$

Specifically, we have for the next sampling time $\left(\sigma=t_{i+1}-t_{i}\right)$ that $\tilde{u}\left(\cdot ; x\left(t_{i+1}\right)\right)$ is a feasible input, hence feasibility at time $t_{i}$ implies feasibility at $t_{i+1}$. Thus, if (2) is feasible for $t=0$, it is feasible for all $t \geq 0$.

Furthermore, if the states for which an admissible input exists converge to the origin, it is clear that the region of attraction $\mathcal{R}$ consists of those points. Convergence: We first show that the value function is decreasing starting from a sampling instant. Remember that the value of $V$ at for $x\left(t_{i}\right)$ is given by:

$$
\begin{aligned}
V\left(x\left(t_{i}\right)\right)=\int_{t_{i}}^{t_{i}+T_{p}} F\left(\bar{x}\left(\tau ; \bar{u}^{\star}\left(\cdot ; x\left(t_{i}\right)\right), x\left(t_{i}\right)\right), \bar{u}^{\star}\left(\tau ; x\left(t_{i}\right)\right) d \tau\right. & \\
& +E\left(\bar{x}\left(t_{i}+T_{p} ; \bar{u}^{\star}\left(\cdot ; x\left(t_{i}\right)\right), x\left(t_{i}\right)\right)\right),
\end{aligned}
$$


and the cost resulting from (6) starting from any $x\left(t_{i}+\sigma ; \bar{u}^{\star}\left(\cdot ; x\left(t_{i}\right)\right), x\left(t_{i}\right)\right)$, $\sigma \in\left(0, t_{i+1}-t_{i}\right]$, using the input $\tilde{u}\left(\tau, x\left(t_{i}+\sigma\right)\right)$, is given by:

$$
\begin{aligned}
& J\left(\tilde{u}\left(\cdot ; x\left(t_{i}+\sigma\right)\right), x\left(t_{i}+\sigma\right)\right)= \int_{t_{i}+\sigma}^{t_{i}+\sigma+T_{p}} \\
& F\left(\bar{x}\left(\tau ; \tilde{u}\left(\cdot ; x\left(t_{i}+\sigma\right)\right), x\left(t_{i}+\sigma\right)\right), \tilde{u}\left(\tau ; x\left(t_{i}+\sigma\right)\right)\right) d \tau \\
&+E\left(\bar{x}\left(t_{i}+\sigma+T_{p} ; \tilde{u}\left(\cdot ; x\left(t_{i}+\sigma\right)\right), x\left(t_{i}+\sigma\right)\right)\right) .
\end{aligned}
$$

Reformulation yields

$$
\begin{aligned}
& J\left(\tilde{u}\left(\cdot ; x\left(t_{i}+\sigma\right)\right), x\left(t_{i}+\sigma\right)\right)=V\left(x\left(t_{i}\right)\right) \\
& \begin{array}{r}
-\int_{t_{i}}^{t_{i}+\sigma} F\left(\bar{x}\left(\tau ; \bar{u}^{\star}\left(\cdot ; x\left(t_{i}\right)\right), x\left(t_{i}\right)\right), \bar{u}^{\star}\left(\tau ; x\left(t_{i}\right)\right)\right) d \tau-E\left(\bar{x}\left(t_{i}+T_{p} ; \bar{u}^{\star}\left(\cdot ; x\left(t_{i}\right)\right), x\left(t_{i}\right)\right)\right) \\
\quad+\int_{t_{i}+T_{p}}^{t_{i}+\sigma+T_{p}} F\left(\bar{x}\left(\tau ; \tilde{u}\left(\cdot ; x\left(t_{i}+\sigma\right)\right), x\left(t_{i}+\sigma\right)\right), \tilde{u}\left(\tau ; x\left(t_{i}+\sigma\right)\right)\right) d \tau \\
+E\left(\bar{x}\left(t_{i}+\sigma+T_{p} ; \tilde{u}\left(\cdot, x\left(t_{i}+\sigma\right)\right), x\left(t_{i}+\sigma\right)\right)\right) .
\end{array}
\end{aligned}
$$

Integrating inequality (5) from $t_{i}+\sigma$ to $t_{i}+\sigma+T_{p}$ starting from $x\left(t_{i}+\sigma\right)$ we obtain zero as an upper bound for the last three terms on the right side. Thus,

$J\left(\tilde{u}\left(\cdot, x\left(t_{i}+\sigma\right)\right), x\left(t_{i}+\sigma\right)\right)-V\left(x\left(t_{i}\right)\right) \leq-\int_{t_{i}}^{t_{i}+\sigma} F\left(\bar{x}\left(\tau ; \bar{u}^{\star}\left(\cdot ; x\left(t_{i}\right)\right)\right), \bar{u}^{\star}\left(\tau ; x\left(t_{i}\right)\right)\right) d \tau$.

Since $\tilde{u}$ is only a feasible but not necessarily the optimal input for $x\left(t_{i}+\sigma\right)$, it follows that

$$
V\left(x\left(t_{i}+\sigma\right)\right)-V\left(x\left(t_{i}\right)\right) \leq-\int_{t_{i}}^{t_{i}+\sigma} F\left(\bar{x}\left(\tau ; \bar{u}^{\star}\left(\cdot ; x\left(t_{i}\right)\right), x\left(t_{i}\right)\right), \bar{u}^{\star}\left(\tau ; x\left(t_{i}\right)\right)\right) d \tau,
$$

i.e. the value function is decreasing along solution trajectories starting at a sampling instant $t_{i}$. Especially we have that:

$$
V\left(x\left(t_{i+1}\right)\right)-V\left(x\left(t_{i}\right)\right) \leq-\int_{t_{i}}^{t_{i+1}} F\left(\bar{x}\left(\tau ; \bar{u}^{\star}\left(\cdot ; x\left(t_{i}\right)\right), x\left(t_{i}\right)\right), \bar{u}^{\star}\left(\tau ; x\left(t_{i}\right)\right)\right) d \tau .
$$

By assumption, this decrease in the value function is strictly positive for $x\left(t_{i}\right) \neq 0$. Since this holds for all sampling instants, convergence holds similarly to $[7,20]$ by an induction argument and the application of Barbalat's lemma.

Various ways to determine a suitable terminal penalty term and terminal region exist. Examples are the use of a control Lyapunov function as terminal penalty $E[25]$ or the use of a local nonlinear or linear control law to determine a suitable terminal penalty $E$ and a terminal region $\mathcal{E}[6,7,9,31,36]$.

Note that Theorem 1 allows to consider the stabilization of systems that can only be stabilized by feedback that is discontinuous in the state [20], e.g. 
nonholonomic mechanical systems. However, for such systems it is in general rather difficult to determine a suitable terminal region and a terminal penalty term.

In the next section, we examine when and under which conditions the nominal NMPC controller is robust against (small) disturbances. The examination is based on the observation that the decrease of the value function in (7) is strictly positive. Since for convergence only a (finite) decrease in the value function is necessary, one can consider the integral term on the right hand side of (7) as a certain robustness margin.

\section{Robustness of State Feedback Sampled-data NMPC}

Several NMPC schemes have been proposed that take uncertainties directly in the controller formulation into account. Typically these schemes follow a game-theoretic approach and require the on-line solution of a min-max problem (e.g. $[8,28,30])$. In this section we do not consider the design of a robustly stable NMPC controller. Instead we examine if sampled-data NMPC based on a nominal model possess certain inherent robustness properties with respect to small model uncertainties and disturbances.

We note that the results derived show similarities to the discrete time results presented in [39]. However, since we consider the stabilization of a continuous time system applying pieces of open-loop input signals, we also have to take the inter-sampling behavior into account. The results are also related to the robustness properties of discontinuous feedback via sample and hold [26]. However, note that we do not consider a fixed input over the sampling time.

Specifically, we consider that the disturbances affecting the system lead to the following modified system equation:

$$
\dot{x}=f(x, u)+p(x, u, w)
$$

where $f, x$ and $u$ are the same as in Section 2, where $p: \mathbb{R}^{n} \times \mathbb{R}^{m} \times \mathbb{R}^{l} \rightarrow \mathbb{R}^{n}$ describes the model uncertainty/disturbance, and where $w \in \mathcal{W} \in \mathbb{R}^{l}$ might be an exogenous disturbance acting on the system. It is assumed that $p$ is bounded over the region of interest, $\mathcal{R} \times \mathcal{U} \times \mathcal{W}$. With regard to existence of solutions, we make the following assumption:

Assumption 1 The system (8) has a continuous solution for any $x(0) \in$ $\mathcal{R}$, any piecewise continuous input $u(\cdot):\left[0, T_{p}\right] \rightarrow \mathcal{U}$, and any exogenous disturbance $w(\cdot):\left[0, T_{p}\right] \rightarrow \mathcal{W}$.

With respect to the value function $V$ we assume that:

Assumption 2 The value function is continuous.

Assumption 3 There exists a $\mathcal{K}$ function $\alpha_{V}$ such that for all $x_{1}, x_{2} \in \mathcal{R}$ : $V\left(x_{1}\right)-V\left(x_{2}\right) \leq \alpha_{V}\left(\left\|x_{1}-x_{2}\right\|\right)$. 
In the following $\Omega_{c}$ denotes level sets of $V$ contained in $\mathcal{R}$, where $c>0$ specifies the level: $\Omega_{c}=\{x \in \mathcal{R} \mid V(x) \leq c\}$. Given this definition we furthermore assume that

Assumption 4 For all compact sets $\mathcal{S} \subset \mathcal{R}$ there is at least one level set $\Omega_{c}$ such that $\mathcal{S} \subset \Omega_{c}$.

In general there is no guarantee that a stabilizing NMPC schemes satisfies Assumption 2, especially if state constrains are present. As is well known [20,34], NMPC can also stabilize systems that cannot be stabilized by feedback that is continuous in the state. Such feedbacks in general also imply a discontinuous value function. Many NMPC schemes, however, satisfy this assumption at least locally around the origin $[7,9,33]$. Furthermore, NMPC schemes that are based on control Lyapunov functions [25] without any constraints on the states and inputs satisfy Assumption 2.

\subsection{Stability Definition and Basic Idea}

We consider persistent disturbances and the repeated application of open-loop inputs, i.e. we cannot react instantaneously to disturbances. Thus, asymptotic stability cannot be achieved, and the region of attraction $\mathcal{R}$ is in general not invariant. As a consequence, we desire in the following only "ultimate boundedness"-results; that the norm of the state after some time becomes small, and that this should hold on inner approximations of $\mathcal{R}$. Furthermore, we want to show that the bound can be made arbitrarily small depending on the bound on the disturbance and the sampling time (practical stability), and that the region of initial conditions where this holds can be made arbitrarily large with respect to $\mathcal{R}$ (semiglobal). In view of Assumption 4 and for simplicity of presentation, we parameterize these regions with level sets.

Specifically we derive bounds that the maximum allowable disturbance and sampling time must fulfill such that we converge from any arbitrary level set of initial conditions $\Omega_{c_{0}} \subset \mathcal{R}$ in finite time to an arbitrary small (but fixed) set $\Omega_{\alpha}$ around the origin without leaving a desired set $\Omega_{c} \subset \mathcal{R}$ with $c>c_{0}$, compare Figure 1 .

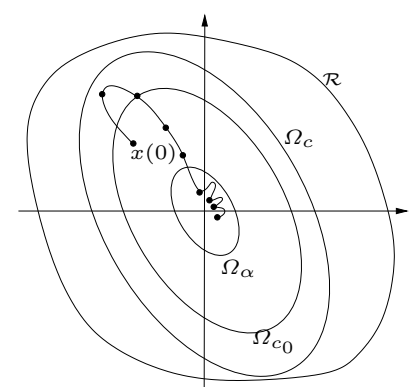

Fig. 1. Set of initial conditions $\Omega_{c_{0}}$, maximum attainable set $\Omega_{c}$, desired region of convergence $\Omega_{\alpha}$ and nominal region of attraction $\mathcal{R}$. 
The derived results are based on the observation that small disturbances and model uncertainties lead to a (small) difference between the predicted

state $\bar{x}$ and the real state $x$. As will be shown, the influence of the disturbance on the value function can be bounded by

$$
\begin{array}{r}
V\left(x\left(t_{i}\right)\right)-V\left(x\left(t_{i+1}\right)\right) \leq-\int_{t_{i}}^{t_{i+1}} F\left(\bar{x}\left(\tau ; \bar{u}^{\star}\left(\cdot ; x\left(t_{i}\right)\right), x\left(t_{i}\right)\right), \bar{u}^{\star}\left(\tau ; x\left(t_{i}\right)\right)\right) d \tau \\
+\epsilon\left(t_{i+1}-t_{i}, x\left(t_{i}\right), w(\cdot)\right)
\end{array}
$$

where $\epsilon$ corresponds to the disturbance contribution. Thus, if the disturbance contribution $\epsilon\left(t_{i+1}\right)$ "scales" with the size of disturbance (it certainly also scales with the sampling time $t_{i+1}-t_{i}$ ) one can achieve contraction of the level sets, at least at the sampling points.

To bound the minimum decrease in the derivations below, we need the following fact:

Fact 1 For any $c>\alpha>0$ with $\Omega_{c} \subset \mathcal{R}, T_{p}>\delta>0$ the lower bound $V_{\min }(c, \alpha, \delta)$ on the value function exists and is non-trivial for all $x_{0} \in \Omega_{c} / \Omega_{\alpha}$ : $0<V_{\min }(c, \alpha, \delta):=\min _{x_{0} \in \Omega_{c} / \Omega_{\alpha}} \int_{0}^{\delta} F\left(\bar{x}\left(s ; u\left(\cdot ; x_{0}\right), x_{0}\right), \bar{u}^{\star}\left(s ; x_{0}\right)\right) d s<\infty$.

\subsection{Additive Disturbances}

Considering the additive disturbance $p$ in (8) we can derive the following Theorem

Theorem 2. Given arbitrary level sets $\Omega_{\alpha} \subset \Omega_{c_{0}} \subset \Omega_{c} \subset \mathcal{R}$. Furthermore, assume that the additive disturbance satisfies $\|p(x, u, w)\| \leq p_{\max }$ with

$$
\alpha_{V}\left(\frac{p_{\max }}{L_{f x}}\left(e^{L_{f x} \bar{\pi}}-1\right)\right) \leq \min \left\{c-c_{0}, V_{\min }(c, \alpha / 4, \underline{\pi}), \alpha / 2\right\}
$$

where $L_{f x}$ is the Lipschitz constant of $f$ over $\Omega_{c}$. Then for any $x(0) \in \Omega_{c_{0}}$ the closed-loop trajectories under the nominal feedback (4) will not leave the set $\Omega_{c}, x\left(t_{i}\right) \in \Omega_{c_{0}} \forall i \geq 0$, and there exists a finite time $T_{\alpha}$ such that $x(\tau) \in \Omega_{\alpha}$ $\forall \tau \geq T_{\alpha}$.

Proof. The proof consists of 3 parts. In the first part we establish conditions that guarantee that the state does not leave the set $\Omega_{c}$ for all $x\left(t_{i}\right) \in \Omega_{c_{0}}$. In the second part we establish conditions such that the states converge in finite time to the set $\Omega_{\alpha / 2}$. In the last part we derive bounds, such that for all $x\left(t_{i}\right) \in \Omega_{\alpha / 2}$ the state does not leave the set $\Omega_{\alpha}$.

First part $\left(x\left(t_{i}+\tau\right) \in \Omega_{c} \forall x\left(t_{i}\right) \in \Omega_{c_{0}}\right)$ : We start by comparing the nominal (predicted) trajectory $\bar{x}$ and the trajectory of the real state $x$ starting from the same initial state $x\left(t_{i}\right) \in \Omega_{c_{0}}$. First note that $x\left(t_{i}+\tau\right)$ and $\bar{x}\left(t_{i}+\tau\right)$ can be written as (skipping the additional arguments the state depends on): 


$$
\begin{aligned}
& x\left(t_{i}+\tau\right)=x\left(t_{i}\right)+\int_{t_{i}}^{t_{i}+\tau}\left(f\left(x(s), u\left(s ; x\left(t_{i}\right)\right)\right)+p\left(x(s), u\left(s ; x\left(t_{i}\right)\right), w(s)\right)\right) d s \\
& \bar{x}\left(t_{i}+\tau\right)=x\left(t_{i}\right)+\int_{t_{i}}^{t_{i}+\tau} f\left(\bar{x}(s), u\left(s ; x\left(t_{i}\right)\right)\right) d s .
\end{aligned}
$$

This is certainly possible for all times $\tau \geq 0$ such that $\bar{x}\left(t_{i}+\tau\right) \in \Omega_{c}$ and $x\left(t_{i}+\tau\right) \in \Omega_{c}$. Subtracting $x$ from $\bar{x}$, using the Lipschitz property of $f$ in $x$ inside of $\Omega_{c}$ (where $L_{f x}$ is the corresponding Lipschitz constant), applying the triangular inequality and partial integration as well as the Gronwall-Bellman inequality we obtain:

$$
\left\|x\left(t_{i}+\tau\right)-\bar{x}\left(t_{i}+\tau\right)\right\| \leq \frac{p_{\max }}{L_{f x}}\left(e^{L_{f x} \tau}-1\right) .
$$

Furthermore, at least as long as $x$ is in $\Omega_{c}$ we have that

$$
\begin{aligned}
V\left(x\left(t_{i}+\tau\right)\right)- & V\left(x\left(t_{i}\right)\right) \leq V\left(x\left(t_{i}+\tau\right)\right)-V\left(\bar{x}\left(t_{i}+\tau\right)\right) \\
& \leq \alpha_{V}\left(\left\|x\left(t_{i}+\tau\right)-\bar{x}\left(t_{i}+\tau\right)\right\|\right) \leq \alpha_{V}\left(\frac{p_{\max }}{L_{f x}}\left(e^{L_{f x} \tau}-1\right)\right) .
\end{aligned}
$$

Here we used that $V\left(\bar{x}\left(t_{i}+\tau\right)\right)-V\left(x\left(t_{i}\right)\right) \leq 0$ (see (7)). Thus, if

$$
\alpha_{V}\left(\frac{p_{\max }}{L_{f x}}\left(e^{L_{f x} \bar{\pi}}-1\right)\right) \leq c-c_{0}
$$

then $x\left(t_{i}+\tau\right) \in \Omega_{c} \tau \in\left[0, t_{i+1}-t_{i}\right], \forall x\left(t_{i}\right) \in \Omega_{c_{0}}$.

Second part $\left(x\left(t_{i}\right) \in \Omega_{c_{0}}\right.$ and finite time convergence to $\left.\Omega_{\alpha / 2}\right)$ : Assume that (12) holds. Note that (12) assures that $x\left(t_{i}+\tau\right) \in \Omega_{c}, \forall \tau \in\left[0, t_{i+1}-t_{i}\right]$. Assuming that $x\left(t_{i}\right) \notin \Omega_{\alpha / 2}$ we know that

$$
\begin{aligned}
V\left(x\left(t_{i+1}\right)\right)-V\left(x\left(t_{i}\right)\right) & =V\left(x\left(t_{i+1}\right)\right)-V\left(\bar{x}\left(t_{i+1}\right)\right)+V\left(\bar{x}\left(t_{i+1}\right)\right)-V\left(x\left(t_{i}\right)\right) \\
& \leq \alpha_{V}\left(\frac{p_{\max }}{L_{f x}}\left(e^{L_{f x} \bar{\pi}}-1\right)\right)-V_{\min }(c, \alpha / 2, \underline{\pi}) .
\end{aligned}
$$

To achieve convergence to the set $\Omega_{\alpha / 2}$ in finite time we need that the right hand side is strictly less than zero. If we require that

$$
\alpha_{V}\left(\frac{p_{\max }}{L_{f x}}\left(e^{L_{f x} \bar{\pi}}-1\right)\right) \leq V_{\min }(c, \alpha / 4, \underline{\pi}),
$$

then we achieve finite convergence, since $V\left(x\left(t_{i+1}\right)\right)-V\left(x\left(t_{i}\right)\right) \leq k_{\mathrm{dec}}:=$ $-V_{\min }(c, \alpha / 2, \underline{\pi})+V_{\min }(c, \alpha / 4, \underline{\pi})<0$ as $\alpha / 4<\alpha / 2$. Thus, for any $x\left(t_{i}\right) \in \Omega_{c_{0}}$ we have finite time convergence to the set $\Omega_{\alpha / 2}$ for a sampling time $t_{m}$ that satisfies $t_{m}-t_{i} \leq T_{\alpha}:=\left\lceil\frac{c-\alpha / 2}{k_{\mathrm{dec}}}\right\rceil$. We can also conclude that $x\left(t_{i+1}\right) \in \Omega_{c_{0}}$ for all $x\left(t_{i}\right) \in \Omega_{c_{0}}$. 
Third part $\left(x\left(t_{i+1}\right) \in \Omega_{\alpha} \forall x\left(t_{i}\right) \in \Omega_{\alpha / 2}\right)$ : This is trivially satisfied following the arguments in the first part of the proof, assuming that

$$
\alpha_{V}\left(\frac{p_{\max }}{L_{f x}}\left(e^{L_{f x} \bar{\pi}}-1\right)\right) \leq \alpha / 2 .
$$

If $V$ is locally Lipschitz over all compact subsets of $\mathcal{R}$, it is possible to replace condition (10) by the following more explicit one:

$$
p_{\max } \leq \frac{L_{f x}}{L_{V}\left(e^{L_{f x} \bar{\pi}}-1\right)} \min \left\{\left(c-c_{0}\right), V_{\min }(c, \alpha / 4, \underline{\pi}), \alpha / 2\right\} .
$$

Here $L_{V}$ is the Lipschitz constant of $V$ over $\Omega_{c}$.

Remark 2. Calculating the robustness bound is difficult, since in general no explicit expression for $V_{\min }(c, \alpha / 4, \underline{\pi})$ can be found, nor it is in general possible to calculate the necessary Lipschitz constants or to obtain an explicit expression for $\alpha_{V}$. The result is still of value, since it underpins that small additive disturbances can be tolerated and it can be utilized for the design of output feedback NMPC schemes.

\subsection{Input Disturbances/Optimization Errors}

The results can be easily extended to disturbances that directly act on the input. To do this we have to assume that $f$ is also Lipschitz in $u$ over $\mathcal{R} \times \mathcal{U}$. One specific case of such disturbances can for example be errors in the optimal input due to the numerical solution of the optimal control problem.

To simplify the presentation we assume that the disturbed input is given by $\bar{u}^{\star}\left(t ; x\left(t_{i}\right)\right)+v(t)$, where $v(\cdot)$ is assumed to be piecewise continuous. Following the ideas in the first part of the proof of Theorem 2, we obtain

$$
\left\|x\left(t_{i}+\tau\right)-\bar{x}\left(t_{i}+\tau\right)\right\| \leq \int_{t_{i}}^{t_{i}+\tau} L_{f x}\|x(s)-\bar{x}(s)\| d s+L_{f u} v_{\max } \tau,
$$

where $L_{f u}$ is the Lipschitz constant of $f(x, u)$ with respect to $u$ over $\Omega_{c} \times \mathcal{U}$, and $v_{\max }$ is the maximum input error. Via the Gronwall-Bellman inequality, this gives (11) with $p_{\max }$ exchanged with $L_{f u} v_{\max }$. The remainder of the proof stays unchanged, thus we obtain the following result for input disturbances:

Theorem 3. Given the level sets $\Omega_{\alpha} \subset \Omega_{c_{0}} \subset \Omega_{c} \subset \mathcal{R}$ and assuming that the additive input disturbance satisfies $\|u\| \leq u_{\max }$ and that

$$
\alpha_{V}\left(\frac{L_{f u} v_{\max }}{L_{f x}}\left(e^{L_{f x} \bar{\pi}}-1\right)\right) \leq \min \left\{\left(c-c_{0}\right), V_{\min }(c, \alpha / 4, \underline{\pi}), \alpha / 2\right\} .
$$

Then for any $x(0) \in \Omega_{c_{0}}$ the closed-loop trajectories under the nominal feedback (4) will not leave the set $\Omega_{c}, x\left(t_{i}\right) \in \Omega_{c_{0}} \forall i \geq 0$, and there exists a finite time $T_{\alpha}$ such that $x(\tau) \in \Omega_{\alpha} \forall \tau \geq T_{\alpha}$. 
Assuming that $V$ is locally Lipschitz we can obtain, similarly as for Theorem 2, a more explicit bound:

$$
p_{\max } \leq \frac{L_{f x}}{L_{f u} L_{V}\left(e^{L_{f x} \bar{\pi}}-1\right)} \min \left\{\left(c-c_{0}\right), V_{\min }(c, \alpha / 4, \underline{\pi}), \alpha / 2\right\} .
$$

One direct implication of this result is that approximated solutions to the optimal control problem can in principle be tolerated. Such approximated solutions can for example result from the numerical integration of the differential equations, as considered in [23]. Furthermore, Theorem 3 gives a theoretical foundation for the so called real-time iteration scheme, in which only one Newton step optimization is performed per sampling instant [13].

Note that the result can, similarly to results on robustness properties of discontinuous feedback via sample-and-hold [26], in principle be expanded to other disturbances, e.g. neglected fast actuator dynamics or computational delays.

\section{Output-Feedback Sampled-data NMPC}

One of the key obstacles for the application of NMPC is that at every sampling instant $t_{i}$ the system state is required for prediction. However, often not all system states are directly accessible. To overcome this problem one typically employs a state observer for the reconstruction of the states. Yet, due to the lack of a general nonlinear separation principle, stability is not guaranteed, even if the state observer and the NMPC controller are both stable.

Several researchers have addressed this question. The approach in [12] derives local uniform asymptotic stability of contractive NMPC in combination with a "sampled" state estimator. In [29], see also [39], asymptotic stability results for observer based discrete-time NMPC for "weakly detectable" systems are given. The results allow, in principle, to estimate a (local) region of attraction of the output feedback controller from Lipschitz constants. In [37] an optimization based moving horizon observer combined with a certain NMPC scheme is shown to lead to (semi-global) closed-loop stability.

Here we follow and expand the ideas derived in $[18,19,24]$, where semiglobal stability results for output-feedback NMPC using high-gain observers are derived. In this section we outline explicit conditions on the observer error, allowing to consider different types of observers such as moving horizon observers, sliding mode observers, observers with a linear error dynamics with arbitrary placeable poles, or observers with a finite time error convergence .

\subsection{Robustness to Estimation Errors}

We assume that instead of the real system state $x\left(t_{i}\right)$ at every sampling instant only a state estimate $\hat{x}\left(t_{i}\right)$ is available. Thus, instead of the optimal feedback (4) the following "disturbed" feedback is applied: 


$$
u\left(\tau ; x\left(t_{i}\right)\right)=\bar{u}^{\star}\left(\tau-t_{i} ; \hat{x}\left(t_{i}\right)\right), \quad \tau \in\left[t_{i}, t_{i+1}\right) .
$$

Note that in principle the estimated state $\hat{x}\left(t_{i}\right)$ can be outside the feasible set $\mathcal{R}$. To avoid feasibility problems we assume that in this case the input is fixed to an arbitrary, bounded value.

Similar to the results in Section 4, we can derive the following result:

Theorem 4. Given the level sets $\Omega_{\alpha} \subset \Omega_{c_{0}} \subset \Omega_{c} \subset \mathcal{R}$. Furthermore, assume that the state estimation error satisfies $\left\|x\left(t_{i}\right)-\hat{x}\left(t_{i}\right)\right\| \leq e_{\max }$, where

$$
\alpha_{V}\left(e^{L_{f x} \bar{\pi}} e_{\max }\right)+\alpha_{V}\left(e_{\max }\right) \leq \min \left\{c-c_{0}, V_{\min }(c, \alpha / 4, \underline{\pi}), \alpha / 2\right\} .
$$

Then for any $x(0) \in \Omega_{c_{0}}$ the closed-loop trajectories with the feedback (4) will not leave the set $\Omega_{c}, x\left(t_{i}\right) \in \Omega_{c_{0}} \forall i \geq 0$, and there exists a finite time $T_{\alpha}$ such that $x(\tau) \in \Omega_{\alpha} \forall \tau \geq T_{\alpha}$.

Proof. Following the ideas of Theorem 2, the proof consists of three parts. However, several adjustments are necessary.

First part $\left(x\left(t_{i}+\tau\right) \in \Omega_{c} \forall x\left(t_{i}\right) \in \Omega_{c_{0}}\right)$ : We consider the difference in the value function between the initial state $x\left(t_{i}\right) \in \Omega_{c_{0}}$ at a sampling time $t_{i}$ and the developing state $x\left(t_{i}+\tau ; x\left(t_{i}\right), u_{\hat{x}}\right)$. For simplicity of notation, $u_{\hat{x}}$ denotes in the following the optimal input resulting from $\hat{x}\left(t_{i}\right)$ and $u_{x}$ the input that correspond to the real state $x\left(t_{i}\right)$. Furthermore, $x_{i}=x\left(t_{i}\right)$ and $\hat{x}_{i}=\hat{x}\left(t_{i}\right)$. By adding and subtracting terms to the difference in the value function, we obtain the following equality

$$
\begin{aligned}
V\left(x\left(\tau ; x_{i}, u_{\hat{x}}\right)\right)-V\left(x_{i}\right)= & V\left(x\left(\tau ; x_{i}, u_{\hat{x}}\right)\right)-V\left(x\left(\tau ; \hat{x}_{i}, u_{\hat{x}}\right)\right) \\
& +V\left(x\left(\tau ; \hat{x}_{i}, u_{\hat{x}}\right)\right)-V\left(\hat{x}_{i}\right)+V\left(\hat{x}_{i}\right)-V\left(x_{i}\right) .
\end{aligned}
$$

The last two terms can be bounded by the continuity assumption on $V$, using the $\mathcal{K}$ function $\alpha_{V}$. Furthermore, note that the third and fourth term start from the same $\hat{x}_{i}$, and that the first term also can be bounded via $\alpha_{V}$ :

$$
\begin{aligned}
& V\left(x\left(\tau ; x_{i}, u_{\hat{x}}\right)\right)-V\left(x_{i}\right) \leq \alpha_{V}\left(e^{L_{f x}\left(\tau-t_{i}\right)}\left\|\hat{x}_{i}-x_{i}\right\|\right) \\
& \quad-\int_{t_{i}}^{\tau} F\left(x\left(s ; \hat{x}_{i}, u_{\hat{x}}\right), u_{\hat{x}}\right) d s+\alpha_{V}\left(\left\|\hat{x}_{i}-x_{i}\right\|\right)
\end{aligned}
$$

From this it follows (since the contribution of the integral is negative) that if

$$
\alpha_{V}\left(e^{L_{f x} \bar{\pi}} e_{\max }\right)+\alpha_{V}\left(e_{\max }\right) \leq c-c_{0}
$$

then $x\left(t_{i}+\tau\right) \in \Omega_{c} \forall \tau \in\left(t_{i+1}-t_{i}\right)$.

Second part $\left(x\left(t_{i}\right) \in \Omega_{c_{0}}\right.$ and finite time convergence to $\left.\Omega_{\alpha / 2}\right)$ : We assume that (17) holds and that $x\left(t_{i}\right) \in \Omega_{c_{0}}$. Note that (12) assures that $x\left(t_{i}+\tau\right) \in \Omega_{c}$, $\forall \tau \in\left[0, t_{i+1}-t_{i}\right]$. Assuming that $x\left(t_{i}\right) \notin \Omega_{\alpha / 2}$ we obtain from (16) that

$$
V\left(x\left(\tau ; x_{i}, u_{\hat{x}}\right)\right)-V\left(x_{i}\right) \leq-V_{\min }\left(c, \alpha / 2, \tau-t_{i}\right)+\alpha_{V}\left(e^{L_{f x} \bar{\pi}\left\|\hat{x}_{i}-x_{i}\right\|}\right)+\alpha_{V}\left(\left\|\hat{x}_{i}-x_{i}\right\|\right)
$$


Similarly to the proof of Theorem 2 we thus know that if

$$
\alpha_{V}\left(e^{L_{f x} \bar{\pi}} e_{\max }\right)+\alpha_{V}\left(e_{\max }\right) \leq V_{\min }(c, \alpha / 4, \underline{\pi})
$$

that we achieve finite time convergence from any $x\left(t_{i}\right) \in \Omega_{c_{0}}$ to the set $\Omega_{\alpha / 2}$ for a sampling time $t_{m}$ that satisfies $t_{m}-t_{i} \leq T_{\alpha}:=\left\lceil\frac{c-\alpha / 2}{k_{\mathrm{dec}}}\right\rceil$. We can also conclude that $x\left(t_{i+1}\right) \in \Omega_{c_{0}}$ for all $x\left(t_{i}\right) \in \Omega_{c_{0}}$.

Third part $\left(x\left(t_{i+1}\right) \in \Omega_{\alpha} \forall x\left(t_{i}\right) \in \Omega_{\alpha / 2}\right)$ : This is trivially satisfied following the arguments in the first part of the proof, assuming that

$$
\alpha_{V}\left(e^{L_{f x} \bar{\pi}} e_{\max }\right)+\alpha_{V}\left(e_{\max }\right) \leq \alpha / 2 .
$$

As for Theorem 3 and Theorem 2 it is possible to derive an explicit bound on $e_{\max }$ assuming that $V$ is locally Lipschitz:

$$
e_{\max } \leq \frac{1}{L_{V}\left(e^{L_{f x} \bar{\pi}}+1\right)} \min \left\{\left(c-c_{0}\right), V_{\min }(c, \alpha / 4, \underline{\pi}), \alpha / 2\right\} .
$$

As outlined in the next subsection, the result allows the design of output feedback NMPC controllers.

\subsection{Output Feedback NMPC}

Theorem 4 lays the basis for the design of observer based output feedback NMPC controllers that achieve semi-global practical stability. Semi-global practical stability here means that for for any given three sets $\Omega_{\alpha} \subset \Omega_{c_{0}} \subset$ $\Omega_{c} \subset \mathcal{R}$ there exists observer parameters and an upper bound on the maximum sampling time $\bar{\pi}$, such that the closed-loop system states will not leave the set $\Omega_{c}$ and converge in finite time to the practical stability region $\Omega_{\alpha}$, where they remain afterwards.

Achieving the semi-global practical stability requires that the observer error $\left\|x\left(t_{i}\right)-\hat{x}\left(t_{i}\right)\right\|$ can be made sufficiently small. Since the required bound of $e_{\max }$ directly depends on $c-c_{0}$ and on $\alpha$, as well as on the maximum $(\bar{\pi})$ and minimum $(\underline{\pi})$ sampling time, using fixed NMPC controller parameters (in addition to the sampling time) requires that the observer has some sort of a tuning knob to decrease the maximum observer error $e_{\max }$.

One possibility for such an observer is a high-gain observer, which allows under certain further restrictions, that the observer error can be sufficiently decreased in a sufficiently short time by increasing the observer gain. This approach has been exploited in $[19,24]$ for output feedback stabilization of nonlinear MIMO systems which are uniformly globally observable. We do not go into details here, and refer to [19] for the sampled-data case.

We note that it is also possible to consider other observers, which allow a sufficient decrease in the observer error. One example are moving horizon observers with contraction constraint [37], where increase of the contraction rate allows to achieve any desired observer error. Other examples are observers 
with linear error dynamics that allows to place the poles of the error dynamics arbitrarily. Such observers can for example be obtained exploiting certain normal forms and output injection [3,27]. Another class of suitable observers are observers that achieve a finite time observer error convergence such as sliding mode observers [14] or the approach presented in $[15,35]$.

\section{Conclusions}

In this paper we considered the stabilization of nonlinear systems using NMPC with sampled measurement information. In a first step we reviewed a generic stability result for sampled-data NMPC. Based on this stability result we considered the inherent robustness properties of sampled-data NMPC. Specifically we showed that NMPC possesses some inherent robustness properties to additive disturbances in the differential equations, to input disturbances and to measurement uncertainties, which could for example be caused by the application of a state observer. The robustness to measurement uncertainty derived here can be used to derive output feedback schemes that achieve semiglobal practical stability, that is, for a fast enough sampling frequency and fast enough observer, it recovers up to any desired accuracy the NMPC state feedback region of attraction (semi-global) and steers the state to any (small) compact set containing the origin (practical stability).

The price to pay is that the value function must be continuous. In general there is no guarantee that nominally stable NMPC schemes satisfy this assumption, especially if constraints on the states are present, see [21]. Thus, future research has to focus on either relaxing this condition, or to derive conditions under which an NMPC scheme does satisfy this assumption, see for example [22].

\section{References}

1. F. Allgöwer, T.A. Badgwell, J.S. Qin, J.B. Rawlings, and S.J. Wright. Nonlinear predictive control and moving horizon estimation - An introductory overview. In P.M. Frank, editor, Advances in Control, Highlights of ECC'99, pages 391-449. Springer, 1999.

2. R.A. Bartlett, A. Wächter, and L.T. Biegler. Active set vs. inerior point strategies for model predicitve control. In Proc. Amer. Contr. Conf., pages 4229-4233, Chicago, Il, 2000.

3. D. Bestle and M. Zeitz. Canonical form observer design for non-linear timevariable systems. Int. J. Contr., 38(2):419-431, 1983.

4. L. Biegler. Efficient solution of dynamic optimization and NMPC problems. In F. Allgöwer and A. Zheng, editors, Nonlinear Predictive Control, pages 219-244. Birkhäuser, 2000.

5. H. Chen. Stability and Robustness Considerations in Nonlinear Model Predictive Control. Fortschr.-Ber. VDI Reihe 8 Nr. 674. VDI Verlag, Düsseldorf, 1997. 
6. H. Chen and F. Allgöwer. Nonlinear model predictive control schemes with guaranteed stability. In R. Berber and C. Kravaris, editors, Nonlinear Model Based Process Control, pages 465-494. Kluwer Academic Publishers, 1998.

7. H. Chen and F. Allgöwer. A quasi-infinite horizon nonlinear model predictive control scheme with guaranteed stability. Automatica, 34(10):1205-1218, 1998.

8. H. Chen, C.W. Scherer, and F. Allgöwer. A robust model predictive control scheme for constrained linear systems. In 5th IFAC Symposium on Dynamics and Control of Process Systems, DYCOPS-5, pages 60-65, Korfu, 1998.

9. W. Chen, D.J. Ballance, and J. O'Reilly. Model predictive control of nonlinear systems: Computational delay and stability. IEE Proceedings, Part D, $147(4): 387-394,2000$.

10. G. De Nicolao, L. Magni, and R. Scattolini. Stability and robustness of nonlinear receding horizon control. In F. Allgöwer and A. Zheng, editors, Nonlinear Predictive Control, pages 3-23. Birkhäuser, 2000.

11. N.M.C. de Oliveira and L.T. Biegler. An extension of Newton-type algorithms for nonlinear process control. Automatica, 31(2):281-286, 1995.

12. S. de Oliveira Kothare and M. Morari. Contractive model predictive control for constrained nonlinear systems. IEEE Trans. Automat. Contr., 45(6):1053-1071, 2000 .

13. M. Diehl, R. Findeisen, S. Schwarzkopf, I. Uslu, F. Allgöwer, H.G. Bock and J. Schlöder. An efficient approach for nonlinear model predictive control of largescale systems. Part I: Description of the methodology. Automatisierungstechnik, 12:557-567, 2002.

14. S. V. Drakunov. Sliding-mode Observer Based on Equivalent Control Method. In Proc. 31st IEEE Conf. Decision Contr., pages 2368-2369, Tucson, 1992.

15. R. Engel and G. Kreisselmeier. A continuous-time observer which converges in finite time. IEEE Trans. Aut. Control, 47(7):1202-1204, 2002.

16. R. Findeisen and F. Allgöwer. The quasi-infinite horizon approach to nonlinear model predictive control. In A. Zinober and D. Owens, editors, Nonlinear and Adaptive Control, Lecture Notes in Control and Information Sciences, pages 89-105, Berlin, 2001. Springer-Verlag.

17. R. Findeisen, M. Diehl, I. Uslu, S. Schwarzkopf, F. Allgöwer, H.G. Bock, J. Schlöder, and Gilles. Computation and performance assesment of nonlinear model predictive control. In Proc. 42th IEEE Conf. Decision Contr., Las Vegas, 2002.

18. R. Findeisen, L. Imsland, F. Allgöwer, and B.A. Foss. Output feedback nonlinear predictive control - a separation principle approach. In Proceedings of 15th IFAC World Congress, Barcelona, Spain, 2002.

19. R. Findeisen, L. Imsland, F. Allgöwer, and B.A. Foss. Output feedback stabilization for constrained systems with nonlinear model predictive control. Int. J. of Robust and Nonlinear Control, 13(3-4):211-227, 2003.

20. F.A. Fontes. A general framework to design stabilizing nonlinear model predictive controllers. Syst. Contr. Lett., 42(2):127-143, 2000.

21. G. Grimm, M.J. Messina, A.R. Teel, and S. Tuna. Examples when model predictive control is nonrobust. submitted, 2002.

22. G. Grimm, M.J. Messina, A.R. Teel, and S. Tuna. Model predictive control: For want of a local control Lyapunov function, all is not lost. submitted, 2002.

23. L. Grüne and D. Nešić. Optimization based stabilization of sampled-data nonlinear systems via their approximate discrete-time models. To appear in SIAM J. Contr. Optim., 2003. 
24. L. Imsland, R. Findeisen, E. Bullinger, F. Allgöwer, and B.A. Foss. A note on stability, robustness and performance of output feedback nonlinear model predictive control. To appear in J. Proc. Contr., 2002.

25. A. Jadbabaie, J. Yu, and J. Hauser. Unconstrained receding horizon control of nonlinear systems. IEEE Trans. Automat. Contr., 46(5):776 -783, 2001.

26. C. Kellett, H. Shim, and A. Teel. Robustness of discontinuous feedback via sample and hold. In Proc. Amer. Contr. Conf., pages 3512-3516, Anchorage, 2002.

27. A.J. Krener and A. Isidori. Linearization by output injection and nonlinear observers. Syst. Contr. Lett., 3:47-52, 1983.

28. S. Lall and K. Glover. A game theoretic approach to moving horizon control. In D. Clarke, editor, Advances in Model-Based Predictive Control. Oxford University Press, 1994.

29. L. Magni, G. De Nicolao, and R. Scattolini. Output feedback and tracking of nonlinear systems with model predictive control. Automatica, 37(10):1601-1607, 2001.

30. L. Magni, H. Nijmeijer, and A.J. van der Schaft. A receding-horizon approach to the nonlinear $\mathrm{H}_{\infty}$ control problem. Automatica, 37(5):429-435, 2001.

31. L. Magni and R. Scattolini. State-feedback MPC with piecewise constant control for continuous-time systems. In Proc. 42th IEEE Conf. Decision Contr., Las Vegas, USA, 2002.

32. F. Martinsen, L.T. Biegler, and B.A Foss. Application of optimization algorithms to nonlinear MPC. In Proceedings of 15th IFAC World Congress, Barcelona, Spain, 2002.

33. D.Q. Mayne, J.B. Rawlings, C.V. Rao, and P.O.M. Scokaert. Constrained model predictive control: stability and optimality. Automatica, 26(6):789-814, 2000.

34. E.S. Meadows, M.A. Henson, J.W. Eaton, and J.B. Rawlings. Receding horizon control and discontinuous state feedback stabilization. Int. J. Contr., 62(5):1217-1229, 1995.

35. P.H. Menold, R. Findeisen, and F. Allgöwer. Finite time convergent observers for linear time-varying systems. Submitted to the 11th Mediterranean Conference on Control and Automation MED'03, 2003.

36. H. Michalska and D.Q. Mayne. Robust receding horizon control of constrained nonlinear systems. IEEE Trans. Automat. Contr., AC-38(11):1623-1633, 1993.

37. H. Michalska and D.Q. Mayne. Moving horizon observers and observer-based control. IEEE Trans. Automat. Contr., 40(6):995-1006, 1995.

38. S.J. Qin and T.A. Badgwell. An overview of industrial model predictive control technology. In J.C. Kantor, C.E. Garcia, and B. Carnahan, editors, Fifth International Conference on Chemical Process Control - CPC V, pages 232-256. American Institute of Chemical Engineers, 1996.

39. P.O.M. Scokaert, J.B. Rawlings, and E.S. Meadows. Discrete-time stability with perturbations: Application to model predictive control. Automatica, 33(3):463470, 1997.

40. M. J. Tenny and J. B. Rawlings. Feasible real-time nonlinear model predictive control. In 6th International Conference on Chemical Process Control - CPC VI, AIChE Symposium Series, 2001.

41. S.J. Wright. Applying new optimization algorithms to model predictive control. In J.C. Kantor, C.E. Garcia, and B. Carnahan, editors, Fifth International Conference on Chemical Process Control - CPC V, pages 147-155. American Institute of Chemical Engineers, 1996. 